\title{
ON SUPPORT POINTS IN THE CLASS OF FUNCTIONS WITH BOUNDED BOUNDARY ROTATION
}

\author{
WALTER HENGARTNER, ALBERT PFLUGER and GLENN SCHOBER \\ Dedicated to Professor Kurt Strebel on the occasion of his sixtieth birthday
}

\section{Introduction}

Let $H(D)$ denote the set of analytic functions in the unit $\operatorname{disc} \boldsymbol{D}=\{|z|<1\}$, endowed with the topology of locally uniform convergence, and let $H(D)^{*}$ be its topological dual. The elements of $H(D)^{*}$ are the continuous linear functionals $L$ on $H(\boldsymbol{D})$. Denoted by $M$ the set of real Baire measures $\mu$ on the unit circle $C=\partial \boldsymbol{D}$ such that $\int_{C} d \mu=2$, and by $M_{k}$ the subset

$$
M_{k}=\left\{\mu: \int_{C} d \mu=2,\|\mu\| \leqq k\right\},
$$

where $\|\mu\|$ is the total variation of $\mu$. Obviously we have $2 \leqq k<\infty$. The formula

$$
f^{\prime}(z)=\exp \int_{|\eta|=1}-\log (1-\eta z) d \mu, \quad f(0)=0,
$$

for the derivative $f^{\prime}$ together with the normalization $f(0)=0$ defines a mapping $V$ of $M$ into $H(D)$, which is injective and continuous in the weak topology of $M$. The representing measure of $f$ is called $\mu$. Discrete measures of $M$ represent the Schwarz-Christoffel mappings; they are dense in $V(M)$.

Because $M_{k}$ is compact in this topology, its image $V\left(M_{k}\right)=V_{k}$ is compact in $H(\boldsymbol{D}) . \quad V_{k}$ is the well-known class of normalized $\left(f(z)=z+a_{2} z^{2}+\ldots\right)$ conformal mappings of $\boldsymbol{D}$ into $\boldsymbol{C}$ having boundary rotation $\pi\|\mu\| \leqq k \pi$. Since the discrete measures $\mu$ with $\|\mu\|=k$ are dense in $M_{k}$, the Schwarz-Christoffel mappings with boundary rotation $k \pi$ are dense in $V_{k}$.

For $2 \leqq k \leqq 4$ the functions in $V_{k}$ are univalent. In particular, $V_{2}$ consists of the normalized univalent mappings of $\boldsymbol{D}$ onto convex domains.

Since $V_{k}$ is compact, the real part of each functional $L$ of $H(D)^{*}$ is maximized within $V_{k}$ by some function, and such functions are called support points if $\operatorname{Re} L$ is not constant on $V_{k}$. It is known that support points of $V_{k}$ are Schwarz-Christoffel mappings with boundary rotation $k \pi$, or equivalently, their representing measures are discrete with norm $k$. This result is in [2], and to provide some framework a 
proof of it will be sketched in Lemma 1. The purpose of this paper is to provide additional properties of support points.

Many functionals of $H(D)^{*}$ have been studied for $V_{k}$. One example is the coefficient functional $a_{n}$ in $f(z)=z+a_{2} z^{2}+\ldots+a_{n} z^{n}+\ldots$. After early attempts it was finally proved in [1] and [3] that for all $n$ the maximum of $\operatorname{Re} a_{n}$ over $V_{k}$ is achieved by the function whose representing measure has its positive and negative part concentrated at $\eta=1$ and at $\eta=-1$, respectively, i.e., for

$$
K(z)=\frac{1}{k}\left(\left(\frac{1+z}{1-z}\right)^{k / 2}-1\right) .
$$

Another example is obtained by evaluation of $f^{\prime}$ at a fixed point $p$ of $\boldsymbol{D} \backslash\{0\}$, or more generally, by $\operatorname{Re}\left\{e^{i \alpha} f^{\prime}(p)\right\}, \alpha \in \boldsymbol{R}$. The extremals for these functionals give the support points of the region $R(p)=\left\{f^{\prime}(p): f \in V_{k}\right\}$, which is convex only for $p$ sufficiently small. However, the set $\left\{\log f^{\prime}(p): f \in V_{k}\right\}$ is convex and is known [7] to have its boundary points furnished by functions $f^{\prime}$ of the form

$$
f^{\prime}(z)=(1-\eta z)^{k / 2-1} /(1-\zeta z)^{k / 2+1}
$$

where $|\zeta|=|\eta|=1$ and $\zeta \neq \eta$. By exponentiation, some of these boundary points are taken onto the support points of $R(p)$. This implies that only functions with derivative of the form (3) can maximize the functionals $\operatorname{Re}\left\{e^{i \alpha} f^{\prime}(p)\right\}$.

Similarly, in Section 5 we shall see that extremals for functionals of the form $\operatorname{Re}\left\{A_{2} a_{2}+A_{3} a_{3}\right\}$, where $A_{2}, A_{3} \in C$ and $a_{2}, a_{3}$ appear in $f(z)=z+a_{2} z^{2}+a_{3} z^{3}+\ldots$, have representing measures supported by only two points. It was among these functionals that we were searching for candidates having extremals of a different type. Since there is none, it seems reasonable to conjecture that all support points of $V_{k}$ must have derivatives of the form (3).

The convex hull of $V_{k}$ will be denoted by co $V_{k}$. It consists of all finite convex combinations of elements of $V_{k}$. Its closure will be denoted by $\overline{c o} V_{k}$. A function $f \in V_{k}$ is an extreme point of co $V_{k}$ or $\overline{c o} V_{k}$ if $f$ cannot be expressed as a proper convex combination of two distinct elements of $\operatorname{co} V_{k}$ or $\overline{\operatorname{co}} V_{k}$, respectively.

It is known [4] that the support points of $V_{2}$ coincide with the extreme points of $\overline{\mathrm{co}} V_{2}$ and are produced by the one-point measures of $M_{2}$. Until now no relations between support points and extreme points were known for $k>2$. For $k \geqq 4$ it was shown in [3] that the extreme points of $\overline{c o} V_{k}$ are functions with derivative of the form (3). For these values of $k$ we shall show that support points of $V_{k}$ have the same form (3). Although we shall give some information for $2<k<4$, a description of the support points of $V_{k}$ and the extreme points of $\overline{\mathrm{co}} V_{k}$ remains as an intriguing open question for $2<k<4$.

In this paper we shall prove the following:

Theorem 1. For $k \geqq 2$ the representing measure of each support point in $V_{k}$ is discrete and has its positive part concentrated at a single point. 
The orem 2. For $k \geqq 4$ each support point of $V_{k}$ has a derivative of the form (3). co $V_{k}$.

Theorem 3. For $2<k<4$ each support point of $V_{k}$ is an extreme point of

Theorem 4. For $k \geqq 2$ each functional $\operatorname{Re}\left\{A_{2} a_{2}+A_{3} a_{3}\right\}, A_{2}, A_{3} \in C \backslash\{0\}$, achieves its maximum within $V_{k}$ only for functions with $f^{\prime}$ of the form (3).

If $A_{3}$ is a positive multiple of $A_{2}^{2}$, then the maximum of $\operatorname{Re}\left\{A_{2} a_{2}+A_{3} a_{3}\right\}$ over $V_{k}$ occurs for a rotation of the function $K(z)$ in (2), namely for $\left(A_{2} /\left|A_{2}\right|\right) K\left(\left|A_{2}\right| z \mid A_{2}\right)$. Some less trivial cases are tabulated in Section 6 where $A_{3}$ is a negative multiple of $A_{2}^{2}$.

\section{The form of support points}

First we sketch a proof of the following important lemma. The framework is basic and will be used later.

Lemma 1. For each support point of $V_{k}$ the representing measure $\mu$ is discrete and has norm $k$.

Proof. Let $f_{0}$ be a support point of $V_{k}$ corresponding to a functional $L$ in $H(D)^{*}$, and let $\mu_{0}$ be the representing measure of $f_{0}$. Then by setting

$$
\mu_{\varepsilon}=\mu_{0}+\varepsilon\left(\mu-\mu_{0}\right), \quad 0 \leqq \varepsilon \leqq 1,
$$

we have a variation of $\mu_{0}$ within $M_{k}$, for each $\mu$ in $M_{k}$. By substituting $\mu_{\varepsilon}$ into (1) we obtain a variation of $f_{0}$ within $V_{k}$, which has the form

$$
f_{\varepsilon}^{\prime}(z)=f_{0}^{\prime}(z)-\varepsilon \int_{C} f_{0}^{\prime}(z) \log (1-\eta z) d\left(\mu-\mu_{0}\right)+O\left(\varepsilon^{2}\right)
$$

as $\varepsilon \rightarrow 0$. Define $L^{\prime}$ by $L^{\prime}\left(f^{\prime}\right)=L(f)$. Then

where

$$
\operatorname{Re} L\left(f_{\varepsilon}\right)=\operatorname{Re} L\left(f_{0}\right)+\varepsilon \int_{C} J(\eta) d\left(\mu-\mu_{0}\right)+O\left(\varepsilon^{2}\right),
$$

$$
J(\eta)=-\operatorname{Re} L^{\prime}\left(f_{0}^{\prime}(z) \log (1-\eta z)\right)
$$

is a harmonic function on $\overline{\boldsymbol{D}}$. The extremality of $f_{0}$ implies that $\int_{C} J(\eta) d\left(\mu-\mu_{0}\right) \leqq 0$ for every $\mu \in M_{k}$. From this it follows that the positive and negative parts of $\mu_{0}$ are supported by subsets of $C$ where $J(\eta)$ reaches its maximum and minimum, respectively. Since $L$ is not constant on $V_{k}$, the function $J(\eta)$ is not constant either, and we conclude that these two subsets of $C$ are discrete and disjoint. This completes the lemma.

Lemma 1 remains true for solutions to nonlinear, Gâteaux differentiable functionals. An earlier proof of the lemma is given in [2]. The discreteness of $\mu$ was shown earlier in [6]. 
We shall also need the following result from [3].

Lemma 2 (Brannan-Clunie-Kirwan). If a function $g$ of $H(D)$ is subordinate to a function $(1+c z) /(1-z)$, for some complex number $c$ with $|c| \leqq 1$, and if $p \geqq 1$. then there is a probability measure $\lambda$ on $C$ such that

$$
g(z)^{p}=\int_{|\xi|=1}\left(\frac{1+c \xi z}{1-\xi z}\right)^{p} d \lambda
$$

Our basic result on the form of support points is the following.

Theorem 1. A support point of $V_{k}$ has a discrete representing measure with positive part supported by a single point. Thus the derivative of a support point is of the form

$$
f^{\prime}(z)=(1-\zeta z)^{-k / 2-1} \prod_{j=1}^{n}\left(1-\eta_{j} z\right)^{-\alpha_{j}}
$$

where $|\zeta|=\left|\eta_{j}\right|=1, \alpha_{j}>0$, and $\sum_{j=1}^{n} \alpha_{j}=k / 2-1$. Moreover, $\zeta$ and $\eta_{1}, \ldots, \eta_{n}$ are mutually disjoint.

Remark. Each support point is a Schwarz-Christoffel mapping with excatly one corner at infinity, where the canonical direction turns around a positive angle of $(k / 2+1) \pi$, while at all other corners it takes negative turns. For $2<k \leqq 4$ the image has an infinite convex complement which is not a strip or a halfplane; for $n=1$ it is a wedge. If $k=2$, the product is deleted and $f(z)=z /(1-\zeta z),|\zeta|=1$ (cf. [4]).

Proof of Theorem 1. Let $f$ maximize the real part of a functional $L$ in $H(D)^{*}$ for which $\operatorname{Re} L$ is nonconstant on $V_{k}$. By Lemma $1 f^{\prime}$ is of the form

$$
f^{\prime}(z)=\prod_{i=1}^{m}\left(1-\zeta_{i} z\right)^{-\beta_{i}} \cdot \prod_{j=1}^{n}\left(1-\eta_{j} z\right)^{\alpha_{j}}
$$

where $\left|\zeta_{i}\right|=\left|\eta_{j}\right|=1, \sum_{j=1}^{n} \alpha_{j}=k / 2-1, \sum_{i=1}^{m} \beta_{i}=k / 2+1$, and where the sets $\left\{\zeta_{i}\right\}$ and $\left\{\eta_{j}\right\}$ are disjoint. Since the function $g(z)=\prod_{i=1}^{m}\left(1-\zeta_{i} z\right)^{-\beta_{i} / \beta}$, with $\beta=k / 2+1$, maps $D$ into the halfplane $\operatorname{Re} w>1 / 2$, it is subordinate to $1 /(1-z)$. As a result, Lemma 2 with $c=0$ implies that $g(z)^{\beta}=\int_{|\xi|=1}(1-\xi z)^{-\beta} d \lambda$ for some probability measure $\lambda$, and by (5) that

where $h(z)=\prod_{j=1}^{n}\left(1-\eta_{j} z\right)^{\alpha_{j}}$.

$$
f^{\prime}(z)=\int_{|\xi|=1} h(z)(1-\xi z)^{-\beta} d \lambda
$$

Let $L^{\prime}$ correspond to $L$ by $L^{\prime}\left(f^{\prime}\right)=L(f)$. Then, by linearity, we have

$$
\operatorname{Re} L^{\prime}\left(f^{\prime}\right)=\int_{|\xi|=1} \varphi(\xi) d \lambda
$$


where $\varphi(\xi)=\operatorname{Re}\left\{L^{\prime}\left(h(z)(1-\xi z)^{-\beta}\right)\right\}$ is harmonic on $\overline{\boldsymbol{D}}$. Since $h(z)(1-\xi z)^{-\beta}$ is the derivative of a function in $V_{k}$, we also have $\varphi(\xi) \leqq \operatorname{Re} L^{\prime}\left(f^{\prime}\right)$ by extremality. As a consequence, the probability measure $\lambda$ must be supported on a subset of $C$ on which $\varphi(\xi)$ achieves its maximum. Since $\varphi(\xi)$ is harmonic on $\bar{D}$, either $\lambda$ has finite support or $\varphi(\xi)$ is constant.

If $\varphi(\xi)$ were constant, we would have $L^{\prime}\left(h(z) z^{\nu}\right)=0$ for every $v=1,2,3, \ldots$ In this case, since $h(z) \neq 0$ in $D$ and $\left(\hat{f}^{\prime} / h\right)(0)=1$, we could conclude that

$$
L^{\prime}\left(\hat{f}^{\prime}\right)=L^{\prime}\left(h \frac{\hat{f}^{\prime}}{h}\right)=L^{\prime}(h)
$$

for all $\hat{f}$ in $V_{k}$. Thus $L$ would be constant on $V_{k}$.

Consequently, the support of $\lambda$ is a finite set $\left\{\xi_{1}, \ldots, \xi_{N}\right\}$. If $\lambda$ has mass $\lambda_{j}$ at $\xi_{j}(j=1, \ldots, N)$, then (5) and (6) imply

$$
\prod_{i=1}^{m}\left(1-\zeta_{i} z\right)^{-\beta_{i}}=\lambda_{1}\left(1-\xi_{1} z\right)^{-\beta}+\ldots+\lambda_{N}\left(1-\xi_{N} z\right)^{-\beta}
$$

Since $\beta=\sum_{i=1}^{m} \beta_{i}$, a comparison of singularities yields $m=N=1$, and the theorem is proved.

\section{Support points for $k \geqq 4$}

For $k \geqq 4$ it was shown in [3] that the extreme points of $\overline{\mathrm{co}} V_{k}$ are functions with derivatives of the form (3). In this section we shall show that even the support points of $V_{k}$ have this form if $k \geqq 4$.

Theorem 2. For $k \geqq 4$ each support point of $V_{k}$ has a derivative of the form

$$
f^{\prime}(z)=(1-\eta z)^{k / 2-1} /(1-\zeta z)^{k / 2+1}
$$

where $|\zeta|=|\eta|=1$ and $\zeta \neq \eta$.

Proof. Let $f$ be a support point corresponding to a functional $L$ of $H(D)^{*}$. According to Theorem $1, f^{\prime}$ satisfies

$$
(1-\zeta z)^{2} f^{\prime}(z)=\prod_{j=1}^{n}\left(\frac{1-\eta_{j} z}{1-\zeta z}\right)^{\alpha_{j}}
$$

where $\zeta, \eta_{1}, \ldots, \eta_{n}$ are distinct points of $C$ and $\alpha=\sum_{j=1}^{n} \alpha_{j}=k / 2-1 \geqq 1$. Each function $\log \left(\left(1-\eta_{j} z\right) /(1-\zeta z)\right)$ maps $\boldsymbol{D}$ onto a horizontal strip of width $\pi$. Therefore, as in [8, Theorem 2.25], the convex sum

$$
s(z)=\sum_{j=1}^{n} \frac{\alpha_{j}}{\alpha} \log \frac{1-\eta_{j} z}{1-\zeta z}
$$

maps $\boldsymbol{D}$ into some horizontal strip of width $\pi$. Thus there exists a constant $c$, where $|c|=1$ and $c \neq-1$, such that $s(z)$ is subordinate to $\log ((1+c z) /(1-z))$. Since 
$e^{s(z)}$ is subordinate to $(1+c z) /(1-z)$ and $\alpha \geqq 1$, Lemma 2 implies that

for some probability measure $\lambda$.

$$
(1-\zeta z)^{2} f^{\prime}(z)=e^{\alpha s(z)}=\int_{|\xi|=1}\left(\frac{1+c \xi z}{1-\xi z}\right)^{\alpha} d \lambda
$$

The next part of the proof runs parallel to the proof of Theorem 1. Let $L^{\prime}$ correspond to $L$ by $L^{\prime}\left(f^{\prime}\right)=L(f)$. Then $\operatorname{Re} L^{\prime}\left(f^{\prime}\right)=\int_{|\xi|=1} \varphi(\xi) d \lambda$ where $\varphi(\xi)=$ $\operatorname{Re}\left\{L^{\prime}\left((1-\zeta z)^{-2}((1+c \xi z) /(1-\xi z))^{\alpha}\right)\right\}$. Since $(1-\zeta z)^{-2}((1+c \xi z) /(1-\xi z))^{\alpha}$ is the derivative of a function in $V_{k}$, we also have $\varphi(\xi) \leqq \operatorname{Re} L^{\prime}\left(f^{\prime}\right)$ by extremality. As a consequence, the probability measure $\lambda$ must be supported on a subset of $C$ on which $\varphi(\xi)$ assumes its maximum. Since $\varphi(\xi)$ is harmonic on $\overline{\boldsymbol{D}}$, either $\lambda$ has finite support or $\varphi(\xi)$ is constant.

If $\varphi(\xi)$ were constant, we would have $L^{\prime}\left((1-\zeta z)^{-2} z^{v}\right)=0$ for every $v=$ $1,2,3, \ldots$, and so

$$
L^{\prime}\left(\hat{f}^{\prime}\right)=L^{\prime}\left((1-\zeta z)^{-2}(1-\zeta z)^{2} \hat{f}^{\prime}(z)\right)=L^{\prime}\left((1-\zeta z)^{-2}\right)
$$

for all $\hat{f}$ in $V_{k}$. Thus $L$ would be constant on $V_{k}$. To make the argument conclusive, we have to establish that no coefficient $c_{v}$ in the expansion of $((1+c z) /(1-z))^{\alpha}$ vanishes. But this follows immediately from Lemma 2 . If $\varepsilon>0$ is sufficiently small, the function $1+\varepsilon z^{v}$ is subordinate to $(1+c z) /(1-z)$, and Lemma 2 implies

$$
0 \neq \alpha \varepsilon=c_{v} \int_{C} \xi^{v} d \tilde{\lambda}
$$

Consequently, the support of $\lambda$ is a finite set $\left\{\xi_{1}, \ldots, \xi_{N}\right\}$, and (7) becomes

$$
f^{\prime}(z)=(1-\zeta z)^{-2} \sum_{i=1}^{N} \lambda_{i}\left(\frac{1+c \xi_{i} z}{1-\xi_{i} z}\right)^{\alpha}
$$

By comparing the singularities in this representation with that from Theorem 1 it follows that $N=1$ and $\xi_{1}=\zeta$. This completes the proof.

\section{Support points for $2<k<4$}

For $k=2$ and $k \geqq 4$ we now know that support points of $V_{k}$ and extreme points of $\overline{c o} V_{k}$ are of the form (3). A similar relation between support points and extreme points for $2<k<4$ would be desirable although nothing is known about extreme points in this case. A slightly weaker statement is given by the following theorem.

Theorem 3. For $2<k<4$ a support point of $V_{k}$ is an extreme point of $\operatorname{co} V_{k}$.

Proof. Let $f$ be a support point of $V_{k}$ and admit a representation as a proper convex combination of two distinct functions $g$ and $h$ in co $V_{k}$. Since $g$ and $h$ are themselves finite convex combinations of functions in $V_{k}$, it follows that $f$ itself is 
a finite convex combination of distinct functions $f_{j}$ in $V_{k}$ :

$$
f=\sum_{j=1}^{N} \lambda_{j} f_{j}, \quad \lambda_{j}>0, \quad \sum_{j=1}^{N} \lambda_{j}=1
$$

It is no loss of generality to assume that all $f_{j}$ differ from $f$.

Since $f$ maximizes the real part of a functional in $H(D)^{*}$, the same is true of each $f_{j}$ in the convex sum. Therefore Theorem 1 implies

$$
f^{\prime}(z)=(1-\zeta z)^{-k / 2-1} \prod_{\sigma}\left(1-\eta_{\sigma} z\right)^{\alpha_{\sigma}}
$$

and

$$
f_{j}^{\prime}(z)=\left(1-\zeta_{j} z\right)^{-k / 2-1} \prod_{\sigma_{j}}\left(1-\eta_{j \sigma_{j}} z\right)^{\alpha_{j \sigma_{j}}}
$$

where $\zeta, \eta_{1}, \ldots, \eta_{n}$ are distinct, $\zeta_{j}, \eta_{j 1}, \ldots, \eta_{j n_{j}}$ are distinct, $|\zeta|=\left|\zeta_{j}\right|=\left|\eta_{\sigma}\right|=\left|\eta_{j \sigma_{j}}\right|=1$, the $\alpha_{\sigma}$ and $\alpha_{j \sigma_{j}}$ are positive, and

$$
\sum_{\sigma} \alpha_{\sigma}=\sum_{\sigma_{j}} \alpha_{j \sigma_{j}}=k / 2-1<1 .
$$

From a comparison of singularities, it follows immediately that $\zeta_{j}=\zeta$ for at least one $j$. By reindexing, we may assume that $\zeta_{1}=\zeta$.

Consider the specific form of the functions $f^{\prime}$ and $f_{j}^{\prime}$ in the identity $f^{\prime}=$ $\sum_{j=1}^{N} \lambda_{j} f_{j}^{\prime}$. Analytic continuation along a small loop around $\bar{\eta}_{\sigma}$ leads to an identity

$$
e^{2 \pi i \alpha_{\sigma}} f^{\prime}(z)=\sum_{j=1}^{N} \lambda_{j} e^{2 \pi i \delta_{j}} f_{j}^{\prime}(z)
$$

for real constants $\delta_{j}$. For $z=0$ this implies

$$
e^{2 \pi i \alpha_{\sigma}}=\sum_{j=1}^{N} \lambda_{j} e^{2 \pi i \delta_{j}}
$$

and is possible only if $e^{2 \pi i \delta_{j}}=e^{2 \pi i \alpha_{\sigma}}, j=1, \ldots, N$. In particular, since $\zeta_{1} \neq \eta_{\sigma}$, the product $\prod_{\sigma_{1}}\left(1-\eta_{1 \sigma_{1}} z\right)^{\alpha_{1 \sigma_{1}}}$ must contain the factor $\left(1-\eta_{\sigma} z\right)^{\alpha}$. At this point we have used the fact that the exponents $\alpha_{\sigma}$ and $\alpha_{1 \sigma_{1}}$ are strictly between 0 and 1 . Since $\sigma$ is arbitrary, the functions $f$ and $f_{1}$ coincide, contrary to assumption, and this completes the proof of the theorem.

Remark. The proof of Theorem 3 does not extend easily to all values of $k$. For example, corresponding to $k=6$ one has $f=f_{1} / 2+f_{2} / 2$ for the functions

$$
\begin{aligned}
& f^{\prime}(z)=\frac{(1-i z)(1+i z)}{(1-\zeta z)^{4}}, \quad f_{1}^{\prime}(z)=\frac{(1-z)^{2}}{(1-\zeta z)^{4}}, \\
& f_{2}^{\prime}(z)=\frac{(1+z)^{2}}{(1-\zeta z)^{4}} .
\end{aligned}
$$

On the other hand, for the values of $k$ not covered by Theorem 3 a stronger conclusion is provided by Theorem 2 . 


\section{Linear combinations of $a_{1}$ and $a_{2}$}

In case $2<k<4$ the preceding results do not say whether the product in (4) contains several distinct factors (splitting) for some support points or whether only one factor occurs for all support points. We shall consider this question for a relatively simple class of functionals. Originally, we had hoped to find examples for splitting in this class, but we shall see that splitting does not occur in this situation.

Let $B_{2}$ be the second coefficient body of $V_{k}$. That is, $B_{2}$ is the set of pairs $\left(a_{2}, a_{3}\right)$ where $a_{2}$ and $a_{3}$ appear as coefficients in the expansion $f(z)=z+a_{2} z^{2}+a_{3} z^{3}+\ldots$ for some function $f$ in $V_{k}$. It is known [7] that $B_{2}$ is homeomorphic to a closed ball in $C^{2}$ and that to each boundary point of $B_{2}$ there corresponds only one function in $V_{k}$. The representing measure $\mu$ of this function is supported by at most 4 points on $C$, where some trigonometric polynomial of degree 2 , say

$$
T\left(e^{i \theta}\right)=\operatorname{Re}\left\{c_{1} e^{i \theta}+c_{2} e^{2 i \theta}\right\},
$$

reaches its maximum and minimum. The positive part of $\mu$ is concentrated at the maxima and the negative part of $\mu$ at the minima. Conversely, each $T$ generates a boundary point of $B_{2}$ in this way. A quantitative description of the boundary of $B_{2}$ has been given also by H. Haario [5].

Now we focus our interest on the support points of $B_{2}$. They maximize over $B_{2}$ the real part of a functional

$$
L=A_{2} a_{2}+A_{3} a_{3}, \quad A_{2}, A_{3} \in C .
$$

By viewing $L$ as a functional on $V_{k}$, it follows from Theorem 1 that functions $f$ corresponding to support points $B_{2}$ must be of the form

$$
f^{\prime}(z)=\left(1-\eta_{1} z\right)^{\alpha_{1}} \cdot\left(1-\eta_{2} z\right)^{\alpha_{2} /(1-\zeta z)^{k / 2+1},}
$$

where $\alpha_{j} \geqq 0, \alpha_{1}+\alpha_{2}=k / 2-1$, and $\zeta \neq \eta_{j}$. That is, the positive part of the representing measure is supported by one point and the negative part by at most two points.

We express $L$ as a function on $M_{k}$ by using the representation (1). It yields

$$
2 a_{2}=\int_{|\eta|=1} \eta d \mu \text { and } 6 a_{3}=\left(2 a_{2}\right)^{2}+\int_{|\eta|=1} \eta^{2} d \mu
$$

By setting $A_{3}=6(a+i b), A_{2}=2(c+i d), a, b, c, d \in \boldsymbol{R}$, and $\eta=e^{i \theta}$, it follows that

$$
\begin{aligned}
\operatorname{Re} L & =a\left(\int \cos 2 \theta d \mu+\left(\int \cos \theta d \mu\right)^{2}-\left(\int \sin \theta d \mu\right)^{2}\right) \\
& -b\left(\int \sin 2 \theta d \mu+2 \int \cos \theta d \mu \cdot \int \sin \theta d \mu\right) \\
& +c \int \cos \theta d \mu-d \int \sin \theta d \mu .
\end{aligned}
$$

Let $\mu_{0}$ maximize $\operatorname{Re} L$ over $M_{k}$, and put

$$
\varrho=2 \int \cos \theta d \mu_{0}, \quad \sigma=2 \int \sin \theta d \mu_{0} .
$$


Applying the $\mu_{\varepsilon}$-variation as considered in the framework of Lemma 1, we obtain

$$
\int_{0}^{2 \pi} J\left(e^{i \theta}\right) d\left(\mu-\mu_{0}\right) \leqq 0 \text { for all } \mu \in M_{k}
$$

as an extremality condition, where now

$$
\begin{aligned}
J\left(e^{i \theta}\right) & =a(\cos 2 \theta+\varrho \cos \theta-\sigma \sin \theta)+c \cos \theta-d \sin \theta \\
& -b(\sin 2 \theta+\varrho \sin \theta+\sigma \cos \theta) .
\end{aligned}
$$

The same argument as used in the proof of Lemma 1 implies that the positive and negative part of $\mu_{0}$ is located at the points of $C$ where $J$ attains its maximum and minimum, respectively. By what was said a few lines earlier, the positive part of $\mu_{0}$ has to be concentrated at a single point while its negative part is supported by at most two points of $C$.

For the remaining part of this investigation we make the assumption that, for our functional $L$, the negative part of $\mu_{0}$ is supported by two distinct points. This assumption will lead us to some necessary conditions on $L$ that provide a contradiction. Therefore we will come to the conclusion that also the negative part of $\mu_{0}$ is supported by a single point.

By assumption the indicator function $J$ must reach its minimum at two distinct points. We shall now characterize such functions $J$. We set $M=\max J$ and $m=\min J$ and apply a lemma of Fejér and F. Riesz saying that a nonnegative trigonometric polynomial is the square of the modulus of an algebraic polynomial in $e^{i \theta}$. Since $M-J$ has a second or fourth order zero, say at $e^{i \theta}$, and is positive elsewhere on $C$, it follows that

$$
M-J\left(e^{i \theta}\right)=p\left|\left(e^{i \theta}-e^{i \alpha}\right)\left(e^{i \theta}-w\right)\right|^{2},
$$

where $w=r e^{i \beta}, 0<r \leqq 1$, and $p>0$. Similarly, we can write

$$
J\left(e^{i \theta}\right)-m=q\left|\left(e^{i \theta}-e^{i \gamma}\right)\left(e^{i \theta}-e^{i \delta}\right)\right|^{2}
$$

with $q>0$ because $J-m$ is positive on $C$ except for two distinct double zeros, say $e^{i \gamma}$ and $e^{i \delta}$. Taking derivatives, we find

and

$$
\begin{gathered}
\frac{\partial J}{\partial \theta}\left(e^{i \theta}\right)=-2 p\left(\left(1+r^{2}\right) \sin (\theta-\alpha)+2 r \sin (\theta-\beta)\right. \\
-2 r \sin (2 \theta-\alpha-\beta))
\end{gathered}
$$

$$
\frac{\partial J}{\partial \theta}\left(e^{i \theta}\right)=4 q(\sin (\theta-\gamma)+\sin (\theta-\delta)-\sin (2 \theta-\gamma-\delta)) .
$$

Since the right hand sides are identical in $\theta$, they remain so if the parameters $\alpha, \beta$, $\gamma, \delta$ are decreased by a constant $\tau$ (that corresponds to replacing $\theta$ by $\theta+\tau$ ). For 
$\tau=(\gamma+\delta) / 2$ we have $\gamma+\delta=0$ without loss of generality. Preserving for the other new parameters the old notations $\alpha$ and $\beta$, we find that equation (12) reduces to

$$
\frac{\partial J}{\partial \theta}\left(e^{i \theta}\right)=-4 q(\sin 2 \theta-\sin (\theta-\gamma)-\sin (\theta+\gamma)) .
$$

By comparing coefficients with the revised (11), it follows that $r p=q, \alpha+\beta=\pi$, $p(1+r)^{2} \sin \alpha=0$, and $2 p(1-r)^{2} \cos \alpha=-4 q \cdot 2 \cos \gamma$. This implies $\sin \alpha=0$, and by a further rotation, we may set $\alpha=0$; hence

$$
\cos \gamma=-\frac{(1-r)^{2}}{4 r} .
$$

This equation restricts the possible values of $r$ to the interval $(3-2 \sqrt{2}, 1]$ and $\cos \gamma$ to $(-1,0]$. Thus, up to a rotation, a three-point support for $\mu_{0}$ is of the following form: The point $\eta=1$ supports the positive part of $\mu_{0}$ and two points $e^{i \gamma}$ and $e^{-i \gamma}$, where $\pi / 2 \leqq \gamma<\pi$, support the negative part of $\mu_{0}$. (Observe that $\gamma=\pi / 2$ is the limiting case corresponding to $r=1$. Here there are two absolute maxima, at $\theta=0$ and $\theta=\pi$, but only one bears the whole mass $k / 2+1$.) The indicator $J$ is then given by

$$
J\left(e^{i \theta}\right)=2 q(\cos 2 \theta-4 \cos \gamma \cdot \cos \theta) .
$$

If $\mu_{0}$ is an extremal measure for the functional $L(f)=A_{2} a_{2}+A_{3} a_{3}$, then $\tilde{\mu}_{0}$ defined by $d \tilde{\mu}_{0}(\theta)=d \mu_{0}(\theta+\tau)$ is an extremal measure for the similar functional $\tilde{L}(f)=A_{2} e^{-i \tau} a_{2}+A_{3} e^{-2 i \tau} a_{3}$. Therefore we may restrict our attention to a functional $L$ having for its extremal measure $\mu_{0}$ the support at $\eta=1$ for the positive part and at $\eta=e^{ \pm i \gamma}$ for its negative part. Comparison of (13) to (10) implies

$$
b=0, \quad a \sigma+d=0, \quad a \varrho+c=-4 \cos \gamma
$$

immediately, and since $q>0$ we may further normalize $a=1$. Thus (8) reduces to

$$
\begin{aligned}
\operatorname{Re} L & =\int \cos 2 \theta d \mu+\left(\int \cos \theta d \mu\right)^{2}-\left(\int \sin \theta d \mu\right)^{2} \\
& +c \int \cos \theta d \mu-d \int \sin \theta d \mu .
\end{aligned}
$$

This functional has to be maximized by a measure $\mu_{0}$ which has its positive part located at $\eta=1$ and its negative part spread over the two points $e^{ \pm i \gamma}$ with weights both nonvanishing.

Let $\operatorname{Re} L=\Phi_{1}+\Phi_{2}$ where

and let

$$
\begin{aligned}
& \Phi_{1}(\mu)=\int \cos 2 \theta d \mu+\left(\int \cos \theta d \mu\right)^{2}+c \int \cos \theta d \mu, \\
& \Phi_{2}(\mu)=-\left(\int \sin \theta d \mu\right)^{2}-d \int \sin \theta d \mu,
\end{aligned}
$$

$$
y(\mu)=\int \sin \theta d \mu .
$$

By (14) and (9) the choice of $\gamma$ within $[\pi / 2, \pi)$ uniquely determines $c, \varrho$, and $\Phi_{1}\left(\mu_{0}\right)$; 
this is because these quantities do not depend on how the negative part of $\mu_{0}$ is spread over $\left\{e^{i \gamma}, e^{-i \gamma}\right\}$. Suppose that the negative part of $\mu_{0}$ has mass $k / 2-1-t$ and $t$ at these points, where $0<t<k / 2-1$. Within this range we have to determine $t$ so that $\Phi_{2}\left(\mu_{0}\right)$ is a maximum under the condition $\sigma+d=0$. Obviously, we have $\Phi_{2}\left(\mu_{0}\right) \leqq d^{2} / 4$ and equality holds if and only if $y\left(\mu_{0}\right)=-d / 2$, in which case the condition $\sigma=-d$ implies $\Phi_{2}\left(\mu_{0}\right)=y\left(\mu_{0}\right)^{2}$. Consequently, because $y\left(\mu_{0}\right)=$ $(2 t+1-k / 2) \sin \gamma$, the expression $\Phi_{2}\left(\mu_{0}\right)$ is maximized only for $t=0$ or $t=k / 2-1$. This contradicts the assumption that the negative part of $\mu_{0}$ is located at two distinct points. Thus we have finally proved that a measure corresponding to a support point of $B_{2}$ has only one negative mass and one positive mass.

Theorem 4. For $k \geqq 2$ each functional $\operatorname{Re}\left\{A_{2} a_{2}+A_{3} a_{3}\right\}, A_{2}, A_{3} \in C \backslash\{0\}$, achieves its maximum within $V_{k}$ only for functions with $f^{\prime}$ of the form (3).

Of course, if $A_{2}$ or $A_{3}$ is zero (but not both), the conclusion of Theorem 4 remains true.

Based on the limited information from Theorem 4 it seems reasonable to. conjecture that support points of $V_{k}$ remain of the form (3) even for $2<k<4$.

\section{The functional $\operatorname{Re}\left\{a_{3}+i \varrho a_{2}\right\}$}

Although Theorem 4 does not actually provide the maximum of $\operatorname{Re}\left\{A_{2} a_{2}+A_{3} a_{3}\right\}$ in closed form, it reduces the problem to an easy one for a small computer. One needs only to find the maximum of a function of two variables.

If $A_{3}$ is a positive multiple of $A_{2}^{2}$, then this maximum occurs for a rotation of the function $K(z)$ in (2). A less trivial example is furnished by the functional

Table for $M=\max _{V_{k}} \operatorname{Re}\left\{a_{3}+i \varrho a_{2}\right\}$

\begin{tabular}{|c|c|c|c|c|c|c|c|c|c|c|c|}
\hline \multirow[b]{2}{*}{$\varrho$} & \multicolumn{2}{|c|}{$k=2$} & \multicolumn{3}{|c|}{$k=3$} & \multicolumn{3}{|c|}{$k=4$} & \multicolumn{3}{|c|}{$k=5$} \\
\hline & $\varphi$ & $M$ & $\varphi$ & $\psi$ & $M$ & $\varphi$ & $\psi$ & $M$ & $\varphi$ & $\psi$ & $M$ \\
\hline 0 & 180 & 1 & 180 & 0 & 1.833 & 180 & 0 & 3 & 180 & 0 & 4.5 \\
\hline 1 & 194.5 & 1.125 & 189.5 & 33.7 & 2.012 & 187.4 & 20.4 & 3.186 & 186.2 & 14.2 & 4.687 \\
\hline 2 & 210.0 & 1.500 & 200.9 & 51.0 & 2.506 & 195.8 & 37.1 & 3.727 & 192.9 & 27.3 & 5.242 \\
\hline 3 & 228.6 & 2.125 & 213.9 & 62.5 & 3.285 & 205.4 & 49.9 & 4.595 & 200.3 & 39.0 & 6.150 \\
\hline 4 & 270 & 3 & 229.8 & 72.3 & 4.341 & 216.5 & 60.2 & 5.773 & 208.7 & 49.2 & 7.395 \\
\hline 5 & 270 & 4 & 256.4 & 84.5 & 5.669 & 230.2 & 69.7 & 7.247 & 218.5 & 58.5 & 8.965 \\
\hline 6 & 270 & 5 & 270 & 90 & 7.167 & 250.9 & 81.0 & 9.014 & 230.3 & 67.4 & 10.849 \\
\hline 7 & 270 & 6 & 270 & 90 & 8.667 & 270 & 90 & 11 & 247.0 & 77.8 & 13.040 \\
\hline 8 & 270 & 7 & 270 & 90 & 10.167 & 270 & 90 & 13 & 270 & 90 & 15.5 \\
\hline$>8$ & 270 & $\varrho-1$ & 270 & 90 & $3 \varrho / 2-11 / 6$ & 270 & 90 & $2 Q-3$ & 270 & 90 & $5 \varrho / 2-9 / 2$ \\
\hline
\end{tabular}


$\operatorname{Re}\left\{a_{3}+i \varrho a_{2}\right\}$ for $\varrho$ real. We computed the maximum value of this functional over $V_{k}$ for several values of $\varrho$ and $k$. There are at most two functions of the form (3) for which the maximum occurs. We have recorded the values $\varphi$ and $\psi$ in degrees at which the positive and negative parts, respectively, of the representing measure $\mu$ are concentrated; that is, $\zeta=\exp i \pi \varphi / 180$ and $\eta=\exp i \pi \psi / 180$. For the second solution one replaces $\varphi$ by $180-\varphi$ and $\psi$ by $180-\psi$.

\title{
References
}

[1] Aharonov, D., and S. Friedland: On an inequality connected with the coefficient conjecture for functions of bounded boundary rotations. - Ann. Acad. Sci. Fenn. Ser. A I Math. 524, 1972, 1-14.

[2] Boutellier, R., and A. Pfluger: Some extremal problems for functions of bounded boundary rotation. - Israel J. Math. 39, 1981, 46-62.

[3] Brannan, D. A., J. G. Clunie, and W. E. Kirwan: On the coefficient problem for functions of bounded boundary rotation. - Ann. Acad. Sci. Fenn. Ser. A I Math. 523, 1973, $1-18$.

[4] Brickman, L., T. H. MacGregor, and D. R. Wilken: Convex hulls of some classical families of univalent functions. - Trans. Amer. Math. Soc. 156, 1971, 91-107.

[5] HaArio, H.: On coefficient bodies of univalent functions. - Ann. Acad. Sci. Fenn. Ser. A I Math. Dissertationes 22, 1978, 1-49.

[6] KirWAN, W. E., and G. Schober: On extreme points and support points for some families of univalent functions. - Duke Math. J. 42, 1975, 285-296.

[7] Pfluger, A.: Functions of bounded boundary rotation and convexity. - J. Analyse Math. 30, 1976, 437-451.

[8] Schober, G.: Univalent functions-Selected topics. - Lecture Notes in Mathematics 478, Springer-Verlag, Berlin-Heidelberg-New York, 1975.

\author{
Université Laval \\ Départment de Mathématiques \\ Québec, G1K 7P4 \\ Canada
}

ETH-Zentrum

Mathematisches Seminar

CH-8092 Zürich

Schweiz

Indiana University

Department of Mathematics

Bloomington, Indiana 47401

USA

Received February 6, 1981 